\title{
ADMISSIBILITY OF MAJORANTS IN CERTAIN MODEL SUBSPACES: NECESSARY CONDITIONS
}

\author{
YU. S. BELOV
}

\begin{abstract}
A nonnegative function $\omega$ on $\mathbb{R}$ is called an admissible majorant for an inner function $\Theta$ if there is a nonzero function $f \in H^{2} \ominus \Theta H^{2}$ such that $|f| \leq \omega$. Some conditions necessary for admissibility are presented in the case where $\Theta$ is meromorphic.
\end{abstract}

\section{$\S 1$. INTRODUCTION}

Let $\Theta$ be an inner function on the upper half-plane $\mathbb{C}^{+}$, and let $K_{\Theta}=H^{2} \ominus \Theta H^{2}$ be the corresponding model subspace of the Hardy space $H^{2}=H^{2}\left(\mathbb{C}^{+}\right)$. Principal attention will be paid to the classical case where $\Theta(z)=e^{i \sigma z}, \sigma>0$; then $K_{\Theta}$ turns into $e^{i \sigma z / 2} P W_{\sigma / 2}$, where $P W_{\sigma}$ denotes the Paley-Wiener space consisting of entire functions of degree at most $\sigma$ that are square integrable on $\mathbb{R}$. A nonnegative function $\omega$ is called an admissible majorant for $K_{\Theta}$ (or a $\Theta$-admissible majorant) if there is a nonzero function $f$ in $K_{\Theta}$ with

$$
|f| \leq \omega \quad \text { a.e. on } \mathbb{R} \text {. }
$$

The class of all $\Theta$-admissible majorants is denoted by $\operatorname{Adm}(\Theta)$.

Conditions sufficient for admissibility were studied in [HM1, HM2, B, BH, BB,, $\mathrm{Bl}$, $\mathrm{Bl3}, \mathrm{MNH}]$. Here we look for conditions necessary for $\Theta$-admissibility in the case where $\Theta$ is a meromorphic Blaschke product on the upper half-plane.

An obvious necessary condition is the convergence of the logarithmic integral $\mathcal{L}(\omega)$ :

$$
\mathcal{L}(\omega):=\int_{\mathbb{R}} \frac{\log \omega(x)}{1+x^{2}} d x>-\infty .
$$

It is well known (see $[\mathrm{BH}]$ ) that this condition is not sufficient. More precisely, for every inner function $\Theta$ there is a nonnegative function $\omega$ such that $\mathcal{L}(\omega)>-\infty$ but $\omega \notin \operatorname{Adm}(\Theta)$. So, $\Theta$-admissibility always requires more than merely the convergence of the logarithmic integral. For instance, in the Beurling-Malliavin theorem pertaining to the case of $\Theta=e^{i \sigma z}, \sigma>0$, some regularity of the majorant $\omega$ is also required.

An arbitrary meromorphic inner function $\Theta$ admits the following representation:

$$
\Theta(z)=e^{i a z} \prod_{k \in \mathbb{Z}} \frac{z-z_{k}}{z-\overline{z_{k}}} \cdot e^{i \alpha_{k}}, \quad \operatorname{Im} z>0,
$$

where $a \geq 0$ and the sequence $\left\{z_{k}\right\}, z_{k}=x_{k}+i y_{k}, x_{k} \in \mathbb{R}, y_{k}>0$, satisfies $\lim _{k \rightarrow \infty}\left|z_{k}\right|=$ $\infty$ and

$$
\sum_{k \in \mathbb{Z}} \frac{y_{k}}{\left|z_{k}\right|^{2}}<+\infty \quad \text { (the Blaschke condition); }
$$

2000 Mathematics Subject Classification. Primary 30D50, 30D55, 30D20.

Key words and phrases. Blaschke product, model subspace, admissible majorant, Beurling-Malliavin theorem.

Supported by RFBR (grant no. 06-01-00313). 
the unimodular factors $e^{i \alpha_{k}}$ ensure the convergence of the infinite product. Let $\arg \Theta$ be a continuous branch of the argument of $\Theta$. Then

$$
\frac{d}{d x} \arg \Theta(x)=a+\sum_{k \in \mathbb{Z}} \frac{2 y_{k}}{\left(x-x_{k}\right)^{2}+y_{k}^{2}}, \quad x \in \mathbb{R}
$$

(see [HM1, p. 1259]).

We shall use the following notation:

$$
\Omega=-\log \omega
$$

$\mathbf{P}$ is the Poisson measure on $\mathbb{R}$ :

$$
d \mathbf{P}(x)=\frac{1}{\pi} \cdot \frac{d x}{1+x^{2}} .
$$

With every function $F \in L^{1}(\mathbf{P})$, we associate its Hilbert transform $\widetilde{F}$ (also denoted sometimes by $h(F))$ :

$$
\widetilde{F}(x)=\frac{1}{\pi} \mathrm{P} . \mathrm{V} \cdot \int_{\mathbb{R}} F(t)\left(\frac{1}{x-t}+\frac{t}{t^{2}+1}\right) d t, \quad x \in \mathbb{R} .
$$

Observe that the integral on the right is finite for a.e. $x \in \mathbb{R}$. The results of the papers HM1, HM2, Bl2, BH] show that the conditions sufficient for admissibility depend on the rate of growth of $\arg \Theta$ (that is, on the right-hand side of (1.1)): the slower $\arg \Theta$ grows, the more narrow is $\operatorname{Adm}(\Theta)$ (as is well known, $K_{\Theta_{2}} \subset K_{\Theta_{1}}$ if and only if $\frac{\Theta_{1}}{\Theta_{2}} \in H^{\infty}$; see $[\mathrm{N}]$ ). In the papers indicated above, the conditions sufficient for admissibility were usually expressed in terms of $\Omega^{\prime}$ or $\widetilde{\Omega}^{\prime}$ (see [HM2, Bl2]). Of course, the first option is preferred because then the conditions are related to $\omega$ itself, whereas in the other case, some additional work is required in order to restate the conditions on $\widetilde{\Omega}$ in terms of $\Omega$.

For a wide class of inner functions $\Theta$, some growth restrictions for $\Omega^{\prime}$ and $\widetilde{\Omega}^{\prime}$ together with the convergence of the logarithmic integral $\mathcal{L}(\omega)$ are sufficient for admissibility. In particular, the Beurling-Malliavin theorem says that an arbitrary majorant $\omega$ such that $\Omega^{\prime}$ is bounded and $\mathcal{L}(\omega)>-\infty$ is admissible in the classical case of $\Theta(z)=e^{i a z}$.

The necessary conditions obtained in the present paper impose restrictions on $\Omega$ itself (or on the averaged function $S_{\lambda}(\Omega)$; see (4.3) for the definition). Nonetheless, these conditions make it possible to construct nonadmissible majorants for which $\Omega^{\prime}$ grows moderately.

The question concerning the sharpness of the sufficient conditions from [HM2, Bl2] remains open. In the last part of the paper, we give some conditions sufficient for admissibility in the case of a meromorphic Blaschke product whose zeros approach $\mathbb{R}$ tangentially. See $[\mathrm{B} 12, \overline{\mathrm{BBH}}]$ for other admissibility results for such inner functions.

The paper is organized as follows. In $\S 2$, we prove several auxiliary lemmas. In $\S 3$, we present some indispensable constructions. A key role in deducing necessary conditions is played by the operator $h_{l}$, the inversion formula (3.4), and equation (2.1). In $\S 4$, the main results are proved. Theorem 4 provides conditions necessary for $\Theta$-admissibility if the argument of $\Theta$ does not grow too fast (specifically, if $\arg \Theta \in L^{1}\left(\left(1+|x|^{l+1}\right)^{-1} d x\right)$, $l \in \mathbb{N}, l>1)$.

The estimates in Theorem 4 depend on the rate of growth of $\arg \Theta$ and on the minimal $l$ for which $\arg \Theta \in L^{1}\left(\left(1+|x|^{l+1}\right)^{-1} d x\right)$. In general, an admissible majorant $\omega$ may take zero values. Thus, the function $\Omega=-\log \omega$ may be infinite at some points. The assumptions of Theorem 4 are fairly bulky, which causes some inconvenience. On the other hand, since $\Omega$ is integrable on the real line against the Poisson measure $\mathbf{P}$, an arbitrary averaging of $\Omega$ is finite on the real line $\left(S_{\lambda}(\Omega)(x)<+\infty, x \in \mathbb{R}\right)$. Theorem 5 provides a condition sufficient for admissibility in terms of $S_{\lambda}(\Omega)$ provided $\arg \Theta$ does not 
grow too fast. Examples $1-3$ show how Theorem 5 applies to certain specific functions $\Omega$ treated in [HM2, Bl2. Statement 3 pertains to a fairly wide class of inner functions with a moderately growing argument (this allows us to apply Theorems 4 and 5). In Subsection 4.4 we shall show that for every inner function with a moderately growing argument, there exists a nonadmissible positive majorant with a convergent logarithmic integral. In $[\mathrm{BH}]$, the existence of a nonnegative nonadmissible majorant with a convergent logarithmic integral was proved for an arbitrary inner function $\Theta$.

In $\S 5$, we show that the necessary conditions are sharp in the classical case (Statement 4) and that there exist nonadmissible majorants for which $\Omega^{\prime}$ grows moderately (see Statements 5 and 6).

In $\S 6$, we study the relationship between certain classes of admissible majorants. This will enable us to obtain conditions sufficient for admissibility in the case of some meromorphic Blaschke products whose zeros tend to the real line tangentially.

\section{§2. Preliminary Remarks}

Let $\Theta$ be an inner function on $\mathbb{C}^{+}$admitting meromorphic continuation to the entire plane $\mathbb{C}$. The following criterion for $\Theta$-admissibility was obtained in HM1.

Theorem 1. The nonnegative function $\omega=e^{-\Omega}$ with $\Omega \in L^{1}(\mathbf{P})$ is $\Theta$-admissible if and only if there exists $m \in L^{\infty}(\mathbb{R})$, an integer-valued function $n$, and a real number $\gamma$ such that $\log m \in L^{1}(\mathbf{P}), m \omega \in L^{2}(\mathbb{R})$, and

$$
\arg \Theta+2 \widetilde{\Omega}=\widetilde{\log m}+2 \pi n+\gamma .
$$

If (2.1) is fulfilled, then $m \omega \in\left|K_{\Theta}\right|=\left\{|f|: f \in K_{\Theta}\right\}$. (We remind the reader that $\arg \Theta$ is a continuous monotone increasing branch of the argument.) We need two lemmas.

Lemma 1. If $m$ and $n$ are as in Theorem 1 , then $n$ is monotone nondecreasing.

Proof. If $m$ and $n$ satisfy (2.1), then there exists an outer function $f$ with

$$
f \in K_{\Theta}, \quad|f|=m \omega \quad \text { a.e. on } \mathbb{R} \text {. }
$$

Since $\Theta$ is meromorphic, the functions in $K_{\Theta}$ are analytic on the real line. Therefore, $f$ possesses only isolated real zeros $a_{k}$. This means that $|f(x)|=\left|x-a_{k}\right|^{p} g, x \in \mathbb{R}$, where $g$ is bounded away from zero in a neighborhood of $a_{k}$. Take a positive function $a \in C^{\infty}(\mathbb{R})$ vanishing off this neighborhood and equal to 1 near $a_{k}$. Then

$$
h(\log |f|)=h(\log (\omega m))=h\left(a p \log \left|x-a_{k}\right|\right)+h(a \log g)+h((1-a) \log |f|) .
$$

The second and third terms on the right are continuous near $a_{k}$. It can easily be observed that the first term jumps down at $a_{k}$. But

$$
2 \pi n=\arg \Theta-2 h(\log (\omega m))
$$

by (2.1), which implies that the function $n$ is monotone nondecreasing, continuous (hence, constant) on any interval $\left(a_{k}, a_{k+1}\right)$ and jumps up at all points $a_{k}$.

Lemma 2. For every positive $\varepsilon$ and $\delta$ and all sufficiently large $x(x>X(\omega, \varepsilon, \delta)>0)$, we have

$$
-|\gamma|-\delta x+\arg \Theta((1-\varepsilon) x) \leq 2 \pi n(x) \leq \arg \Theta((1+\varepsilon) x)+\delta x+|\gamma|
$$

( $\gamma$ is the number occurring in (2.1)). 
Proof. Consider the intervals $I_{x, \varepsilon}=[x,(1+\varepsilon) x]$. We observe that $\frac{\varepsilon}{|x|}>\mathbf{P}\left(I_{x, \varepsilon}\right)>\frac{\varepsilon}{2|x|}$ for $\varepsilon$ sufficiently small and $|x|>2$. By the Kolmogorov theorem, if $f \in L^{1}(\mathbf{P})$, then

$$
P(x:|h(f)(x)|>a)=o(1 / a), \quad a \rightarrow+\infty .
$$

Consequently, for every $f \in L^{1}(\mathbf{P}), \sigma>0$, and $x>X(f, \sigma, \varepsilon)$ we have

$$
|h(f)(s)| \leq \sigma s
$$

for some $s \in I_{x, \varepsilon}$. In particular, this is true for $f=-\log (\omega m)$.

To prove the upper estimate for $n$, we observe that, for $x>X(f, \sigma, \varepsilon)$, Lemma 1 implies

$$
\begin{aligned}
2 \pi n(x) & \leq 2 \pi n(s)=\arg \Theta(s)+2 f(s)-\gamma \\
& \leq \arg \Theta((1+\varepsilon) x)+2 \sigma(1+\varepsilon) x+|\gamma|<\arg \Theta((1+\varepsilon) x)+\delta x+|\gamma|,
\end{aligned}
$$

provided $\sigma<\frac{\delta}{2(1+\varepsilon)}$; i.e., we have arrived at the right-hand part of (2.2). To prove the left-hand part, we consider intervals $I_{-x, \varepsilon}=[(1-\varepsilon) x, x]$.

Remark 1. It is easy to prove similar estimates of $n$ for $x<0$, i.e.,

$$
-|\gamma|-\delta|x|+\arg \Theta((1+\varepsilon) x) \leq 2 \pi n(x) \leq \arg \Theta((1-\varepsilon) x)+\delta|x|+|\gamma|, \quad x<0 .
$$

\section{§3. Generalized Hilbert transformation. Inversion formula}

We shall need the Poisson measures $\mathbf{P}_{l}$ on $\mathbb{R}, l=1,2, \ldots$ :

$$
d \mathbf{P}_{l}(x)=\frac{d x}{1+|x|^{l+1}} .
$$

Then $\mathbf{P}_{1}$ is proportional to $\mathbf{P}$.

By Theorem 1, a majorant $\omega$ is $\Theta$-admissible if and only if equation (2.1) is solvable. We rewrite (2.1) as follows:

$$
\arg \Theta-2 \pi n-\gamma=2 h(\log (\omega m)) .
$$

To deduce conditions necessary for admissibility, it would be desirable to take the Hilbert transforms of the two sides of (3.1) in order to release $\log \omega$ from the operator $h$, and obtain a "pure" formula for $\omega$ in terms of $\arg \Theta, m$, and $n$. However, generally speaking, $\arg \Theta-n+\gamma \notin L^{1}(\mathbf{P})$. Therefore, we need a new version of the classical inversion formula for the Hilbert transformation. The following theorem is a consequence of a result due to V. I. Smirnov (see, e.g., Ba]).

Theorem 2. If $u \in L^{1}(\mathbf{P})$ and $h(u) \in L^{1}(\mathbf{P})$, then

$$
h(h(u))=-u+\text { const } \quad \text { a.e. on } \mathbb{R} \text {. }
$$

In general, $h(u) \notin L^{1}(\mathbf{P})$ for $u \in L^{1}(\mathbf{P})$; moreover, $h(u)$ may fail to belong even to $\bigcup_{l \in \mathbb{N}} L^{1}\left(\mathbf{P}_{l}\right)$. If $u \in L^{1}(\mathbf{P})$, then $h(h(u))$ can be understood as the so-called $A$-integral, but, as far as I know, the validity of (3.2) is still a problem (see Ba]). Our problem is even more involved because we are interested in an inversion formula for $u \in L^{1}\left(\mathbf{P}_{l}\right)$ with $l$ arbitrary. To overcome the difficulties, we begin with the following definition: for $u \in L^{1}\left(\mathbf{P}_{l}\right)$, put

$$
h_{l}(u)(x)=\frac{1}{\pi} \mathrm{P} \cdot \mathrm{V} \cdot \int_{\mathbb{R}} u(t) \frac{1}{x-t} \cdot \operatorname{Re}\left(\frac{x+i}{t+i}\right)^{l} d t .
$$

Note that $h_{1}=h$. 
3.1. Inversion formula for $h_{l}$. Before proving an inversion formula for the operator $h_{l}$, we establish a simple property of it: $h_{l}(p)=0$ for every polynomial $p$ of degree strictly smaller than $l$.

Proof. By the Cauchy formula for the upper half-plane, we have

$$
p(z)=\frac{(z+i)^{l}}{2 \pi i} \int_{\mathbb{R}} \frac{p(t)}{(t+i)^{l}(t-z)} d t, \quad \operatorname{Im} z>0 .
$$

The Sokhotskiu-Privalov formula shows that

$$
\frac{p(x)}{2}=\frac{(x+i)^{l}}{2 \pi i} \mathrm{P} . \mathrm{V} \cdot \int_{\mathbb{R}} \frac{p(t)}{(t-x)(t+i)^{l}} d t
$$

for all $x \in \mathbb{R}$. Taking the imaginary part on the right, we see that $h_{l}(p)=0$ for every real polynomial $p$ with $\operatorname{deg} p<l$.

For convenience, we shall treat $h(u)$ modulo a summand $p$, where $p$ is an arbitrary polynomial of degree smaller than $l$.

We pass to an analog of (3.2) for $h_{l}$.

Theorem 3. Suppose $v \in L^{1}(\mathbf{P})$ is a real-valued function with $h(v) \in L^{1}\left(\mathbf{P}_{l}\right)$. Then

$$
h_{l}(h(v))=-v+p_{v} \quad \text { a.e. on } \mathbb{R},
$$

where $p_{v}$ is a polynomial with $\operatorname{deg} p<l$.

Proof. Consider the following operator $h_{l}^{*}$ :

$$
h_{l}^{*}(u)(x)=\frac{1}{\pi} \mathrm{P} . \mathrm{V} \cdot \int_{\mathbb{R}} u(t) \frac{1}{x-t} \cdot\left(\frac{x+i}{t+i}\right)^{l} d t, \quad u \in L^{1}\left(\mathbf{P}_{l}\right) .
$$

Clearly, $\operatorname{Re} h_{l}^{*}(u)=h_{l}(u)$. At the same time,

$$
\begin{aligned}
\operatorname{Im} h_{l}^{*}(u)(x) & =\frac{1}{\pi} \mathrm{P} \cdot \mathrm{V} \cdot \int_{\mathbb{R}} u(t) \frac{1}{x-t} \cdot \operatorname{Im}\left(\frac{x+i}{t+i}\right)^{l} d t \\
& =\frac{1}{\pi} \mathrm{P} \cdot \mathrm{V} \cdot \int_{\mathbb{R}} u(t) \frac{1}{x-t} \cdot \operatorname{Im}\left(\frac{x t+1}{t^{2}+1}-i \frac{x-t}{t^{2}+1}\right)^{l} d t .
\end{aligned}
$$

It is easily seen that the function $\frac{1}{x-t} \cdot \operatorname{Im}\left(\frac{x t+1}{t^{2}+1}-i \frac{x-t}{t^{2}+1}\right)^{l}$ is a polynomial of degree smaller than $l$ in $x$. Consequently,

$$
h_{l}^{*}(u)=h_{l}(u)+i p_{u},
$$

where $p_{u}$ is a polynomial with $\operatorname{deg} p<l$. If $u \in L^{1}(\mathbf{P})$ and $h_{1}^{*}(u) \in L^{1}(\mathbf{P})$, then an application of Theorem 2 yields

$$
h_{1}^{*}\left(h_{1}^{*}(u)\right)=-u+\text { const a.e. on } \mathbb{R} \text {. }
$$

We shall also need a formula relating $h_{l}^{*}(u)$ and $h_{1}^{*}(u)$. It is readily seen that

$$
h_{l}^{*}(u)-h_{1}^{*}(u)=\frac{1}{\pi} \mathrm{P} . \mathrm{V} \cdot \int_{\mathbb{R}} u(t) \frac{1}{x-t} \cdot\left(\frac{(x+i)^{l-1}}{(t+i)^{l-1}}-1\right) \frac{x+i}{t+i} d t=Q_{u}(x) .
$$

Observe that $Q_{u}$ is a polynomial of degree at most $l-1$. Consequently,

$$
h_{l}^{*}(u)=h_{1}^{*}(u)+Q_{u} .
$$

Suppose that $u \in L^{1}\left(\mathbf{P}_{l}\right), l \in \mathbb{N}$. Then

$$
h_{l}^{*}(u)(x)=\frac{1}{\pi} \mathrm{P} . \mathrm{V} \cdot \int_{\mathbb{R}} u(t) \frac{1}{x-t} \cdot\left(\frac{x+i}{t+i}\right)^{l} d t=(x+i)^{l-1} h_{1}^{*}\left(\frac{u(t)}{(t+i)^{l-1}}\right) .
$$


We know that $h_{1}(v) \in L^{1}\left(\mathbf{P}_{l}\right)$. Therefore, by (3.5), we obtain $h_{1}^{*}(v) \in L^{1}\left(\mathbf{P}_{l}\right)$. Using (3.6) and (3.7), we arrive at

$$
\begin{aligned}
h_{l}^{*}\left(h_{1}^{*}(v)\right)(x) & =h_{l}^{*}\left(h_{l}^{*}(v)\right)-h_{l}^{*}\left(Q_{v}\right)=(x+i)^{l-1} h_{1}^{*}\left(\frac{h_{l}^{*}(v)(t)}{(t+i)^{l-1}}\right)-h_{l}^{*}\left(Q_{v}\right) \\
& =(x+i)^{l-1} h_{1}^{*}\left(h_{1}^{*}\left(\frac{v(t)}{(t+i)^{l-1}}\right)\right)-h_{l}^{*}\left(Q_{v}\right) \\
& =-v+\mathrm{const}-h_{l}^{*}\left(Q_{v}\right) .
\end{aligned}
$$

A direct calculation shows that $h_{l}^{*}\left(Q_{v}\right)$ is a polynomial of degree at most $l-1$. Now, (3.4) can be deduced from (3.5).

3.2. Some estimates of $h_{l}(f)$. If $f$ vanishes near zero, then there exists a polynomial $p_{f}$ with $\operatorname{deg} p_{f} \leq l-1$ and such that

$$
h_{l}(f)(x)=\frac{1}{\pi} \mathrm{P} \cdot \mathrm{V} \cdot \int_{\mathbb{R}} f(t) \frac{x^{l}}{t^{l}(x-t)} d t+p_{f} .
$$

On the other hand, if $f$ is compactly supported, then

$$
\begin{aligned}
\left|\pi h_{l}(f)\right| & =\mid \text { P.V. } \int_{\mathbb{R}} \frac{f(t)}{x-t} d t+p_{f} \mid \\
& =\mid \text { P.V. } \int_{\mathbb{R}} \frac{f(t)-f(x)}{x-t} d t+p_{f} \mid \preceq\left\|f^{\prime}\right\|_{\infty}+\left(1+|x|^{l-1}\right),
\end{aligned}
$$

where $\operatorname{deg} p_{f} \leq l-1$. Therefore, $\left|h_{l}(f)(x)\right| \preceq(1+|x|)^{l-1}, x \in \mathbb{R}$.

We shall need another estimate of $h_{l}(f)$.

Statement 1. Suppose that $f \in L^{1}\left(\mathbf{P}_{l}\right) \cap C^{1}(\mathbb{R})$. Then there exists a polynomial $p_{f}$ of degree at most $l$ and such that

$$
\begin{aligned}
\left|h_{l}(f)(x)\right| \leq & \frac{1+\delta}{\delta}|x|^{l} \int_{|t| \geq(1+\delta)|x|} \frac{|f(t)|}{|t|^{l+1}} d t+\int_{1<|t|<(1+\delta)|x|}|f(t)| \frac{|x|^{l-1}}{|t|^{l}} d t \cdot l(1+\delta)^{l} \\
& +2|f(t)|_{-(1+\delta)|x|}^{(1+\delta)|x|} \log |x||+| \int_{|t|<(1+\delta)|x|} \log |x-t| d f(t)|+| p_{f}(|x|) \mid
\end{aligned}
$$

for every $\delta>0$.

Proof. Take a function $a \in C^{\infty}(\mathbb{R})$ supported on $[-2,2]$ and such that $a \equiv 1$ on $[-1,1]$. Then $h_{l}(f)=h_{l}(a f)+h_{l}((1-a) f)$. Since $h_{l}(a f) \preceq(1+|x|)^{l-1}$ and $(1-a(x)) f(x)=f(x)$ for $|x|>2$, we may assume that $f \equiv 0$ on $[-1,1]$. Consequently,

$$
\begin{aligned}
\pi h_{l}(f)(x) & =\mathrm{P} \cdot \mathrm{V} \cdot \int_{\mathbb{R}} f(t) \frac{x^{l}}{t^{l}(x-t)} d t+p_{f} \\
& =\int_{|t| \geq(1+\delta)|x|}+\int_{|t|<(1+\delta)|x|}+p_{f}=I_{1}+I_{2}+p_{f},
\end{aligned}
$$

where $p_{f}$ is a polynomial of degree at most $l$. It is easily seen that

$$
\left|I_{1}\right| \leq|x|^{l} \int_{|t| \geq(1+\delta)|x|}|f(t)| \frac{d t}{|t|^{l}|x-t|} \leq \frac{1+\delta}{\delta}|x|^{l} \int_{|t| \geq(1+\delta)|x|} \frac{|f(t)|}{|t|^{l+1}} d t .
$$

On the other hand,

$$
\left|I_{2}\right| \leq \mid \text { P.V. } \int_{|t|<(1+\delta)|x|} f(t) \frac{d t}{x-t}\left|+\int_{|t|<(1+\delta)|x|}\right| f(t)|| \frac{1}{t^{l}} \cdot \frac{x^{l}-t^{l}}{x-t} \mid d t .
$$


Since $\left|\frac{x^{l}-t^{l}}{x-t}\right| \leq \sum_{k=0}^{l-1}|x|^{l-1-k}|t|^{k} \leq l(1+\delta)^{l}|x|^{l-1}$ for $|t| \leq(1+\delta)|x|$, we obtain

$$
\int_{|t|<(1+\delta)|x|}|f(t)|\left|\frac{1}{t^{l}} \cdot \frac{x^{l}-t^{l}}{x-t}\right| d t \leq \int_{1<|t|<(1+\delta)|x|}|f(t)| \frac{|x|^{l-1}}{|t|^{l}} d t \cdot l(1+\delta)^{l} .
$$

Next,

$$
\begin{aligned}
\mid \text { P.V. } & \int_{|t|<(1+\delta)|x|} f(t) \frac{d t}{x-t} \mid \\
& =\left|\int_{|t|<(1+\delta)|x|} \log \right| x-t|d f(t)+(f(t) \log |x-t|)|_{-(1+\delta)|x|}^{(1+\delta)|x|} \mid .
\end{aligned}
$$

The claim follows from (3.10)-(3.13).

Remark 2. Estimate (3.9) is true also in the case where $f$ is a step function, i.e., $f=c_{k}$ on $\left(a_{k}, a_{k}+1\right)$, where $\left\{a_{k}\right\}$ and $\left\{c_{k}\right\}$ are monotone increasing sequences and $\left\{a_{k}\right\}$ has no limit points.

Proof. All the details of the preceding proof remain the same, except for the fourth integral in (3.9), which is a finite sum of terms of the form DP.V. $\int_{B}^{C} \frac{d t}{x-t}=$ $D(\log |x-C|-\log |x-B|)$, where $D=c_{k}, B=a_{k}, C=a_{k+1}($ or $\pm(1+\delta) x)$.

Remark 3. We can use (3.9) for the differences $f_{1}-f_{2}$, where $f_{1}$ is as in Statement 1 and $f_{2}$ is as in Remark 2 .

Put $j_{x, \delta}=\{t \in \mathbb{R}:|t| \leq(1+\delta)|x|\}$.

Remark 4. Suppose $f \in C^{1}(\mathbb{R})$ is monotone decreasing. Then

$$
\left|\int_{j_{x, \delta}} \log \right| x-t|d f(t)| \leq 2 \max _{[x-1, x+1]}\left|f^{\prime}\right|+\left(\Delta_{j_{x, \delta}} f\right) \cdot \log ((2+\delta)|x|)
$$

for every $x \in \mathbb{R}$, where $\Delta_{j_{x, \delta}} f$ is the increment of $f$ on the interval $j_{x, \delta}$.

Proof. Indeed,

$$
\left|\int_{j_{x, \delta}}\right| \leq\left|\int_{[x-1, x+1]}+\int_{j_{x, \delta} \backslash[x-1, x+1]}\right|=\left|J_{1}+J_{2}\right|
$$

Next,

$$
\begin{aligned}
& J_{1} \leq 2 \max _{[x-1, x+1]}\left|f^{\prime}\right| \int_{0}^{1}|\log u| d u=2 \max _{[x-1, x+1]}\left|f^{\prime}\right|, \\
& J_{2} \leq \int_{j_{x, \delta} \backslash[x-1, x+1]} \log (|x|+(1+\delta|x|)) d f(t)=\log ((2+\delta)|x|) \cdot\left(\Delta_{j_{x, \delta}} f\right) .
\end{aligned}
$$

\section{§4. Conditions neCESSARY FOR ADMissibility}

Now, we are ready to prove the main technical result of the paper. It gives a condition necessary for $\Theta$-admissibility and appears fairly bulky. But we shall see subsequently that, still, it is a source of more natural (and even sharp) admissibility conditions. We return to a meromorphic inner function $\Theta$ and put $A\left(=A_{\Theta}\right)=\arg \Theta$ for short.

Theorem 4. Suppose that $A \in L^{1}\left(\mathbf{P}_{l}\right)$ for some natural number $l$, and that $\omega=e^{-\Omega}$ (where $\Omega \geq 0$ ) is a $\Theta$-admissible function. Then for every $\delta>0$ there exists a constant $C(\delta, l)$ such that

$$
\Omega(x) \leq C(\delta, l)(I+J+S)
$$


where

$$
\begin{aligned}
& I=I_{A}=|x|^{l} \int_{|t| \geq|x|} \frac{|A(t)|}{|t|^{l+1}} d t+\int_{1<|t|<(1+2 \delta)|x|}|A(t)| \frac{|x|^{l-1}}{|t|^{l}} d t, \\
& J=\Delta_{j_{x, \delta}} A \cdot|\log | x||+\sup _{[x-1, x+1]}\left|A^{\prime}\right|+|x|^{l-1}+1, \\
& S=\left|\sum_{\left|a_{k}\right|<(1+\delta)|x|} \log \right| x-a_{k}||,
\end{aligned}
$$

and the real sequence $\left\{a_{k}\right\}$ satisfies

$$
\operatorname{card}\left\{k:\left|a_{k}\right|<|x|\right\} \leq \Delta_{j_{x, 2 \delta}} A+C_{1}|x|+C_{2}
$$

for some constants $C_{1}$ and $C_{2}$.

Proof. Since $\omega$ is $\Theta$-admissible, there exist functions $m$ and $n$ resolving equation (2.1). Therefore,

$$
\arg \Theta-2 \pi n-\gamma=2 h(\log (\omega m)),
$$

where $0 \leq m \leq 1$. There is no loss of generality in assuming that $n$ vanishes near zero. By our assumptions on $A$ and by Lemma 2, the left-hand side of (4.2) is in $L^{1}\left(\mathbf{P}_{l}\right)$. Thus,

$$
h_{l}(\arg \Theta-2 \pi n+\gamma)=h_{l}\left(h_{1}(\log (\omega m))\right)=-\log \omega-\log m+p_{\omega} \geq \omega+p_{\omega},
$$

where $p_{\omega}$ is a polynomial with $\operatorname{deg} p_{\omega}<l$. Put $g=A-2 \pi n$. Using Lemma 2, we obtain the inequality $|g(x)| \leq 2|A((1+\varepsilon) x)|+C_{1}|x|+C_{2}$; the function $g$ satisfies the assumptions of Remark 3. Consequently, we may use Statement 1 to estimate $\left|h_{l}(g)\right|$. Taking $(1+\varepsilon) t$ for a new variable, we see that

$$
K_{1}=\int_{|t| \geq(1+\delta)|x|} \frac{|A((1+\varepsilon) t)|}{|t|^{l+1}} d t \leq 2 \int_{|t| \geq|x|} \frac{|A(t)|}{|t|^{l+1}} d t
$$

if $\varepsilon$ is sufficiently small. The integral $\int_{1<|t|<(1+\delta)|x|}|A((1+\varepsilon) t)| \frac{|x|^{l-1}}{|t|^{l}} d t=K_{2}$ is estimated similarly. Next,

$$
\int_{|t|<(1+\delta|x|)} \log |x-t| d g=\int \log |x-t| d A(t)-\int \log |x-t| d(2 \pi n(t))=K_{3}-K_{4} .
$$

Using Remark 4 (with $f=A$ ), we estimate $K_{3}$. At the same time, $S=\left|K_{4}\right|=$ $\left|\sum_{\left|a_{k}\right|<(1+\delta)|x|} \log \right| x-a_{k}||$, where the $a_{k}$ are the points of discontinuity of $n$. Since the sequence $\left\{a_{k}\right\}$ has no limit points, we see that $n$ is monotone increasing by Lemma 1. In fact, $n$ may have a jump whose height is an arbitrary natural number, which corresponds to repetition of members of $\left\{a_{k}\right\}$. By Lemma 2, the sequence $\left\{a_{k}\right\}$ possesses the required properties if $\varepsilon$ is sufficiently small. Collecting the inequalities for $K_{1}, K_{2}$, $K_{3}$, and $K_{4}$, we arrive at the desired estimate.

4.1. Admissibility and estimates for certain averagings of $\Omega$. In Theorem 3 , the function $\Omega$ was estimated in terms of an expression with logarithmic singularities at the points $a_{k}$. Therefore, it is more convenient to consider some averagings of $\Omega$ rather than $\Omega$ itself. For such averagings, we shall obtain upper estimates in terms of the function $A$, avoiding the use of $\left\{a_{k}\right\}$. Let $\lambda$ be a function defined on $\mathbb{R}$ and taking values in $(0,1]$. We put

$$
S_{\lambda}(f)(x)=\frac{1}{2 \lambda(x)} \int_{|s-x|<\lambda(x)} f(s) d s, \quad x \in \mathbb{R} .
$$

Now, we can exhibit some conditions in terms of $S_{\lambda}(\Omega)$ that are necessary for admissibility. 
The next lemma prepares certain estimates for $S_{\lambda}(\Omega)$. We put $I=[m, m+h], m \in \mathbb{R}$, $0<h \leq 1$.

Lemma 3. There is a universal constant $c>0$ such that

$$
\frac{1}{h} \int_{I}|\log | u|| d u \leq c \cdot \max \{|\log m|,|\log h|, 1\} .
$$

Corollary 1. Suppose that $\lambda$ is a function defined on $\mathbb{R}$ and satisfying $\lambda(\mathbb{R}) \subset(0,1]$. Put $S_{\lambda}^{a}(x):=\frac{1}{2 \lambda(x)} \int_{x-\lambda(x)}^{x+\lambda(x)}|\log | t-a|| d t, a \in \mathbb{R}$. Then

$$
S_{\lambda}^{a}(x) \leq c \cdot \max \{\log (|x|+|a|+1),|\log | \lambda(x)||, 1\},
$$

where $c$ is a universal constant.

Proof. Putting $u=a-t$, we reduce the claim to Lemma 3 (with $m=x-a-\lambda(x)$, $h=2 \lambda(x))$.

We remind the reader that $A$ stands for $\arg \Theta$.

Theorem 5. Let a meromorphic inner function $\Theta$ satisfy $A \in L^{1}\left(\mathbf{P}_{l}\right)$, where $l \in \mathbb{N}$. If the function $\omega=e^{-\Omega}(\Omega \geq 0)$ is $\Theta$-admissible, then for every $\delta>0$ there exists a constant $C(\delta, l)$ such that

$$
S_{\lambda}(\Omega)(x) \leq C(\delta, l)\left(S_{\lambda}(I+J)+\Delta_{j_{x, \delta}} A \cdot \max \{|\log | x||,|\log | \lambda(x)||, 1\}\right), \quad x \in \mathbb{R},
$$

where the quantities $I(=I(A, l, \delta))$ and $J(=J(A, \delta, l))$ are the same as in Theorem 4.

Proof. We put $\delta_{0}=\delta / 2$ and apply Theorem 4. This results in the estimate $|\Omega(x)| \leq$ $C(\delta, l)(I+J+S)$. Lemma 3 implies the following inequality:

$$
S_{\lambda}(|\log | x-a||) \leq c \cdot \max \{|\log | x||,|\log \lambda(x)|, 1\},
$$

where $|a|<2|x|$ and $c$ is a universal constant. Let $n$ be the counting function for the sequence $\left\{a_{k}\right\}$, i.e., $n(x)=\operatorname{card}\left\{a_{k}: 0<a_{k}<x\right\}$ for $x \geq 0$, and $n(x)=-\operatorname{card}\left\{a_{k}\right.$ : $\left.x<a_{k}<0\right\}$ for $x<0$. Next,

$$
S_{\lambda}(\Omega) \leq C(\delta, l)\left(S_{\lambda}(I+J)+S_{\lambda}(S)\right) .
$$

On the other hand,

$$
\begin{aligned}
S_{\lambda}(S) & \leq \sum_{\left|a_{k}\right|<\left(1+\delta_{0}\right)|x|} S_{\lambda}\left(|\log | x-a_{k}||\right) \\
& \leq c \Delta_{j_{x, \delta}} n \cdot \max \{|\log | x||,|\log \lambda(x)|, 1\} .
\end{aligned}
$$

Applying Lemma 2 (with an appropriate $\varepsilon$ ) to $n$, we prove the claim.

4.2. Examples. We give several examples of applications of Theorem 5 .

Example 1. Put $\Theta(z)=e^{i \sigma z}(\sigma>0)$. Then every $\Theta$-admissible majorant $\omega=e^{-\Omega}$ satisfies the inequality

$$
\left|S_{1}(\Omega)(x)\right| \leq C_{1}|x \log | x||+C_{2}, \quad x \in \mathbb{R} .
$$

Proof. Note that $A(x)=\sigma x \in L^{1}\left(\mathbf{P}_{2}\right)$. Applying Theorem $4\left(l=2, \delta=\frac{1}{2}, \lambda \equiv 1\right)$, we see that

$$
\left|S_{1}(\Omega)(x)\right| \leq C(\delta, l)\left(S_{\lambda}(I+J)+|x \log | x||+1\right) .
$$

It is easily seen that

$$
I=|x|^{2} \int_{|t| \geq|x|} \frac{\sigma d t}{t^{2}}+\int_{1<|t|<2|x|} \frac{\sigma|x+t|}{t} d t \preceq|x \log | x||+1 .
$$

Clearly, $J \preceq|x \log | x||+1$. Consequently, $S_{1}(I+J) \preceq|x \log | x||+1$. 
Example 2. Let $f: \mathbb{R} \rightarrow \mathbb{R}$ be a strictly monotone increasing differentiable function, and let $B_{f}$ be the Blaschke product with the zeros $z_{k}=f(k)+i, k \in \mathbb{Z}$. Suppose that $\lim _{k \rightarrow \pm \infty} f(k)= \pm \infty, \int_{\mathbb{R}} \frac{d t}{f^{2}(t)+1}<\infty$ (this ensures the convergence of the Blaschke product) and that $\left(f^{-1}\right)^{\prime} \in \operatorname{Lip}_{\beta}(\mathbb{R}), 0<\beta<1$. If a function $\omega=e^{-\Omega}$ is $B_{f}$-admissible, then for every $\delta>0$ there are constants $C, C_{1}, C_{2}$ such that

$$
\left|S_{1}(\Omega)(x)\right| \leq S_{1}\left(I_{f^{-1}}+C \Delta_{j_{x, \delta}} f^{-1} \cdot|\log | x||\right)+C_{1}|x|+C_{2}, \quad x \in \mathbb{R},
$$

where $I$ is the same as in Theorem $5\left(\right.$ with $\left.A=f^{-1}\right)$.

Proof. We observe that $f^{-1}-n=O(1)$, where $n$ is the counting function of the sequence $\{f(k)\}$. It is well known that $B_{f}^{\prime}=2 \pi\left(f^{-1}\right)^{\prime}+O(1)$ (see Theorem 2.4). Consequently, $\left|\arg B_{f}(x)\right| \preceq\left|f^{-1}(x)\right|+|x|$. Since $\left(f^{-1}\right)^{\prime} \in \operatorname{Lip}_{\beta}(\mathbb{R})$, we see that $\left(f^{-1}\right)^{\prime}(x)=o(x)$. Applying Theorem $4(l=2)$, we prove the claim.

The following example is a particular case of Example 2.

Example 3. Let $B_{\alpha}$ be the Blaschke product with the zeros $z_{k}=\operatorname{sgn}(k)|k|^{\alpha}+i\left(\frac{1}{2}<\right.$ $\alpha<1$ ). If the function $\omega=e^{-\Omega}$ is $B_{\alpha}$-admissible, then there exist constants $C_{1}$ and $C_{2}$ such that

$$
\left|S_{1}(\Omega)(x)\right| \leq C_{1}|x|^{\frac{1}{\alpha}}|\log | x||+C_{2}, \quad x \in \mathbb{R} .
$$

Proof. We apply the estimate in Example $2\left(f(x)=|x|^{1 / \alpha},|x|>1\right)$. It can easily be shown that $S_{1}\left(I_{f^{-1}}\right) \preceq|x|^{\frac{1}{\alpha}}+1$. Indeed,

$$
I_{f^{-1}} \preceq I_{|x|^{1 / \alpha}}=|x| \int_{|t| \geq|x|} \frac{|t|^{1 / \alpha}}{|t|^{3}} d t+\int_{1<|t|<2|x|}|t|^{1 / \alpha} \frac{|x|}{|t|^{2}} d t \preceq|x|^{1 / \alpha}+1 .
$$

Observe that $\Delta_{j_{x, \delta}} f^{-1} \preceq|x|^{1 / \alpha}$.

For convenience, we have estimated the function $S_{1}(\Omega)$ in Examples 2 and 3. It can easily be seen that the same estimates are true for every function $\lambda$ with $\lambda(x) \succeq \frac{1}{1+|x|}$, because $\max (|\log | x||,|\log | \lambda(x)||) \preceq \log |x|, x>2$.

Let $(\arg B)^{\prime}(x) \asymp(1+|x|)^{k-1}$, where $k \in(0,1)$. Then the condition $S_{1}(\Omega)(x) \preceq$ $|x|^{k} \log |x|,|x|>10$, is necessary for admissibility. In particular, the majorants of the form $\omega_{\gamma}(x)=\exp \left(-|x|^{\gamma}\right)$ are not admissible if $\gamma>k$. At the same time, the results of HM2] imply that $\omega_{\gamma}$ is $B$-admissible for $\gamma<k$.

4.3. Blaschke products subject to the condition $\arg B \in L^{1}\left(\mathbf{P}_{l}\right)$. The next two statements describe the arrangements of zeros of a meromorphic Blaschke product $B$ that ensure the inclusion $\arg B \in L^{1}\left(\mathbf{P}_{l}\right)$ (so that the results of Subsection 4.1 become applicable).

Statement 2. Let $B$ be the Blaschke product with zeros $z_{k}=x_{k}+i y_{k} \in \mathbb{C}^{+}$, where the sequence $z_{k}$ possesses the following properties:

$$
\text { 1) } \left.\lim _{k \rightarrow+\infty}\left|z_{k}\right|=+\infty ; \quad 2\right) \sum_{k \in \mathbb{Z}} \frac{y_{k}}{\left|z_{k}\right|^{2}}<+\infty .
$$

Denote by $n_{*}$ the counting function for the sequence $\left\{x_{k}\right\}$. Then for every $\delta \in(0,1)$ there exist constants $C_{1}, C_{2}$ such that

$$
|\arg B(x)|<\pi\left|n_{*}((1+\delta) x)\right|+C_{1}|x|+C_{2} .
$$


Proof. Let $x>0$. We observe that, under the agreement $B(0)=0$, we have

$$
\arg B(x)=\int_{0}^{x} \arg B^{\prime}(s) d s=\int_{0}^{x}\left(\sum_{k \in \mathbb{Z}} \frac{y_{k}}{\left(s-x_{k}\right)^{2}+y_{k}^{2}}\right) d s .
$$

Now,

$$
\sum_{x_{k}<0} \frac{y_{k}}{\left(s-x_{k}\right)^{2}+y_{k}^{2}} \leq \sum_{x_{k}<0} \frac{y_{k}}{x_{k}^{2}+y_{k}^{2}}
$$

and moreover, the contribution of the summands with negative $x_{k}$ to $\arg B(x)$ does not exceed $C x$ for some constant $C$. Next,

$$
\sum_{x_{k} \geq 0}=\sum_{x_{k} \geq 0,\left|x-x_{k}\right| \leq \delta x_{k}}+\sum_{x_{k} \geq 0,\left|x-x_{k}\right|>\delta x_{k}}=\Sigma_{1}+\Sigma_{2} .
$$

For every $s \in[0, x]$, we have

$$
\begin{aligned}
\operatorname{card}\left\{k:\left|s-x_{k}\right| \leq \delta x_{k}\right\} & \leq \operatorname{card}\left\{k:(1-\delta) x_{k}<s\right\} \\
& \leq \operatorname{card}\left\{k: x_{k}<x /(1-\delta)\right\}=n_{*}(x /(1-\delta)),
\end{aligned}
$$

whereas $\int_{0}^{x} \frac{y_{k}}{\left(s-x_{k}\right)^{2}+y_{k}^{2}} d s \leq \int_{\mathbb{R}} \frac{y_{k}}{\left(s-x_{k}\right)^{2}+y_{k}^{2}} d s=\pi$, and

$$
\Sigma_{1} \leq \pi n_{*}\left(\frac{x}{1-\delta}\right)
$$

But

$$
\Sigma_{2} \leq \int_{0}^{x} \frac{y_{k}}{\delta^{2} x_{k}^{2}+y_{k}^{2}} d s \leq \frac{1}{\delta^{2}} \sum_{k \in \mathbb{Z}} \frac{y_{k}}{x_{k}^{2}+y_{k}^{2}} \cdot x .
$$

The proof for negative $x$ is similar.

Statement 2 relates the growth of $A=\arg \Theta$ to the counting function $n_{*}$. In particular, if $n_{*} \in L^{1}\left(\mathbf{P}_{l}\right), l>1$, then $A(=\arg \Theta) \in L^{1}\left(\mathbf{P}_{l}\right)$. On the other hand, there exist subsets $D_{l}$ of the upper half-plane $\mathbb{C}^{+}$with the following property: if all zeros of a Blaschke product $B$ belong to $D_{l}$, then $\arg B \in L^{1}\left(\mathbf{P}_{l}\right)$. For example, if $\inf _{k} \operatorname{Im} z_{k}>0$, where $\left\{z_{k}\right\}$ is the sequence of zeros of $B$, then $\arg B \in L^{1}\left(\mathbf{P}_{2}\right)$.

For every positive integer $l$, we define a domain $D_{l} \subset \mathbb{C}^{+}$as follows:

$$
D_{l}=\left\{z=x+i y: y>\min \left(|x|^{2-l}, 1\right)\right\} .
$$

Statement 3. Suppose that a sequence $\left\{z_{k}\right\} \subset \mathbb{C}^{+}$satisfies the Blaschke condition and lies in $D_{l}, l>1$. If $B$ is the Blaschke product with zeros $\left\{z_{k}\right\}$, then $A \in L^{1}\left(\mathbf{P}_{l}\right)$.

Proof. Put $z_{k}=x_{k}+i y_{k}$. Then $\sum_{k} \frac{y_{k}}{x_{k}^{2}+y_{k}^{2}}<+\infty$ (the Blaschke condition). We consider the terms of the sequence $\left\{z_{k}\right\}$ with $y_{k} \geq x_{k}$ and introduce the Blaschke product with these zeros. From the convergence condition for Blaschke products, it easily follows that $\sum_{k: y_{k} \geq x_{k}} \frac{1}{y_{k}}<+\infty$. Therefore,

$$
\left(\arg B_{1}\right)^{\prime}=\sum_{k: y_{k} \geq x_{k}} \frac{y_{k}}{\left(x-x_{k}\right)^{2}+y_{k}^{2}} \leq \sum_{k: y_{k} \geq x_{k}} \frac{1}{y_{k}} \preceq 1 .
$$

Accordingly, $\left|\arg B_{1}(x)\right| \preceq|x|+1$, whereas $A \in L^{1}\left(\mathbf{P}_{2}\right)$. So, we may assume that the domain $\left\{z: z \in \mathbb{C}^{+}, \operatorname{Im} z>\operatorname{Re} z\right\}$ contains no terms of the sequence. Let $n_{*}$ be a counting function for the sequence $\left\{x_{k}\right\}$ such that $n_{*}=0$ near zero. Since $x_{k}>y_{k}$ and the series $\sum_{k} \frac{y_{k}}{x_{k}^{2}+y_{k}^{2}}$ converges, the series $\sum_{k} \frac{y_{k}}{x_{k}^{2}}$ also converges. Then

$$
\sum_{k} \frac{y_{k}}{x_{k}^{2}} \geq \sum_{k} \frac{1}{\left|x_{k}\right|^{l}}=\int_{\mathbb{R}} \frac{d n_{*}(t)}{|t|^{l}}=l \int_{\mathbb{R}} \frac{\left|n_{*}(t)\right|}{|t|^{l+1}} d t .
$$

Consequently, $n_{*} \in L^{1}\left(\mathbf{P}_{l}\right)$. Using Statement 2, we obtain the claim. 
If the zeros of a Blaschke product belong to $D_{l}$ with some $l>1$, we can use Theorem 5 and 6 to estimate a $\Theta$-admissible function $\Omega$ from above. For example, if all zeros lie on the line $\mathbb{R}+i$, we can put $l=2$.

4.4. An outline of the construction of nonadmissible majorants. In this subsection we show that Theorems 5 can provide some specific examples of nonadmissible majorants for functions $\Theta$ with tempered growth of the $\operatorname{argument} A=\arg \Theta$. Among these examples, there are functions $\Omega$ integrable against the Poisson measure (i.e., $\Omega \in L^{1}(\mathbf{P})$ ). The existence of such functions $\Omega$ was proved in $[\mathrm{BH}]$ for all inner functions $\Theta$, but the construction there was less explicit, and the resulting functions $\omega$ had many zeros on the real line, whereas the method described here makes it possible to obtain functions bounded away from zero on any compact segment. We remind the reader (see the Introduction) that an arbitrary specific nonadmissible function $\omega$ yields a uniqueness theorem for $K_{\Theta}$.

For an inner function $\Theta$ with $A=\arg \Theta \in L^{1}\left(\mathbf{P}_{l}\right)$, we introduce the following notation:

$$
\begin{aligned}
& F(x):=I(x)+J(x)+C A(2|x|) \cdot \log (|x|+1)+C|x|, \\
& G(x):=C A(2|x|), \quad x \in \mathbb{R},
\end{aligned}
$$

where $I=I_{A}$ and $J=J_{A}$ were defined in Theorem $4(\delta=1 / 2)$. We emphasize that $F$ and $G$ depend only on $\Theta$ (more precisely, on $A$ ). As usual, $\lambda: \mathbb{R} \rightarrow(0,1]$ is a function determining an averaging operator $S_{\lambda}$ (see (4.3)). Finally, we put $h:=$ $F+G \cdot(|\log (\lambda)|+1)$. Theorem 4 implies the following statement.

Corollary 2. If $\limsup _{x \rightarrow+\infty} \frac{S_{\lambda}(\Omega)(x)}{h(x)}=+\infty$, then the function $\omega=e^{-\Omega}$ is not $\Theta$ admissible. (Surely, the condition with lim $\sup _{x \rightarrow+\infty}$ can be replaced by a similar one with $\left.\lim \sup _{x \rightarrow-\infty}\right)$.

This result makes it possible to construct specific nonadmissible majorants. Let $\left\{x_{k}\right\}_{k \in \mathbb{N}}$ be a positive sequence tending to $+\infty$. We put $I_{k}=\left(x_{k}-\lambda\left(x_{k}\right), x_{k}+\lambda\left(x_{k}\right)\right)$. Consider an arbitrary sequence $\left\{B_{k}\right\}_{k \in \mathbb{N}}$ of positive numbers tending to $+\infty$. If

$$
\left.\Omega\right|_{I_{k}} \geq \frac{B_{k}}{2} \cdot h\left(x_{k}\right)
$$

for every $k \in \mathbb{N}$, then the function $\omega:=e^{-\Omega}$ is not $\Theta$-admissible by Corollary 2. Next, condition (4.5) is compatible with the integrability of $\Omega$ against the Poisson measure. Indeed, let $x_{k+1}-x_{k}>2$ for all $k \in \mathbb{N}$, so that the intervals $I_{k}$ are mutually disjoint. We choose $\lambda$ in such a way that the function $h \cdot \lambda$ is bounded on $\mathbb{R}$ (we recall that the functions $F$ and $G$ are continuous and, therefore, locally bounded). Next, we put

$$
\Omega=\sum_{k \in \mathbb{N}} \frac{B_{k}}{2} \cdot h\left(x_{k}\right) \cdot \chi_{I_{k}} .
$$

Clearly, (4.5) is true for all $k \in \mathbb{N}$. At the same time, $\mathbf{P}\left(I_{k}\right) \preceq \frac{B_{k}}{x_{k}^{2}}$. If the series $\sum_{k} \frac{B_{k}}{x_{k}^{2}}$ converges, then $\Omega$ is integrable against the Poisson measure. Indeed,

$$
\int \Omega d \mathbf{P} \leq \mathrm{const} \sum_{k} B_{k} \cdot h\left(x_{k}\right) \cdot \lambda\left(x_{k}\right) \cdot x_{k}^{-2} \leq \mathrm{const} \sum_{k} \frac{B_{k}}{x_{k}^{2}} .
$$

We can take an arbitrary unbounded sequence $B_{k}$ with $\sum_{k} \frac{B_{k}}{k^{2}}<+\infty$, because $x_{k}>2 k$. The resulting nonadmissible majorant $\omega=e^{-\Omega}$ can be expressed in terms of $A=\arg \Theta$. 


\section{$\S 5$. Sharpness of NECESSARY CONDITIONS}

In this section we present some examples illustrating the sharpness of Theorems 4 and 5. We start with the classical case of the Paley-Wiener spaces and show that the result described in Example 1 is sharp to a great extent.

Statement 4. Put $\Theta(z)=e^{i \sigma z}(\sigma>0)$. Then for every monotone decreasing positive function $\gamma$ with $\lim _{x \rightarrow+\infty} \gamma(x)=0$ there exists a $\Theta$-admissible function $\omega=e^{-\Omega}$ and a sequence $\left\{x_{n}\right\}_{1}^{\infty}, x_{n} \rightarrow+\infty$, such that

$$
\left|S_{1}(\Omega)\left(x_{n}\right)\right| \geq x_{n} \gamma\left(x_{n}\right) \log x_{n}
$$

(Recall that $S_{1}$ is the averaging operator; see (4.3).)

Proof. Without loss of generality, we may assume that $\gamma(x)>1000 / x$ for $x>1$ (we can replace $\gamma$ with $\gamma+1000 / x$ for such an $x$ ). Take a sequence $\left\{\delta_{n}\right\}_{n \in \mathbb{N}}$ in $[0,1]$ such that $\sum_{n} \delta_{n}<\sigma$. We put

$$
f(z)=\prod_{n \in \mathbb{N}}\left[\cos \left(\frac{z}{2^{n}}\right)\right]^{\left[2^{n} \delta_{n}\right]} .
$$

The product converges uniformly on compact subsets of $\mathbb{C}$ because $\cos \left(\frac{z}{2^{n}}\right) \asymp 1-z^{2} / 2^{2 n}$, $n \rightarrow+\infty$. Next, we choose an infinite set $\mathbb{N}^{*} \subset \mathbb{N}$ and put $\delta_{n}=0$ for $n \in \mathbb{N} \backslash \mathbb{N}^{*}$, $\delta_{n}=C \gamma\left(x_{n}\right)$ for $n \in \mathbb{N}^{*}$, where $x_{n}:=\frac{\pi}{2} 2^{n}, n \in \mathbb{N}$, and $C$ is an appropriate constant. We observe that $f$ is an entire function of finite type at most $\sigma$. Indeed,

$$
|f(z)|=\prod_{n \in \mathbb{N}}\left|\cos \left(\frac{z}{2^{n}}\right)\right|^{\left[2^{n} \delta_{n}\right]} \leq \prod_{n} \exp \left(\frac{\operatorname{Im} z}{2^{n}}\left[2^{n} \delta_{n}\right]\right) \leq \exp \left(\operatorname{Im} z \sum_{n} \delta_{n}\right) .
$$

Moreover, $f$ is bounded on the real line. If $\alpha$ is a zero of $f$, then $\frac{f(z)}{z-\alpha} \in L^{2}(\mathbb{R})$ and $\frac{f(z)}{z-\alpha} \in P W_{\sigma}$. Therefore, $|f|$ is an admissible majorant for $K_{\Theta}$. Put $\Omega:=-\log |f|$. A drawback of this definition is that $\Omega$ has infinite values (so, $\omega$ has zero values). This can be eliminated by addition of $i \varepsilon_{n}$ to the argument of the $n$th factor, where $\left\{\varepsilon_{n}\right\}$ is a positive sequence rapidly tending to zero. But this would have complicated the forthcoming simple estimates, so we leave things as they are. Next,

$$
\Omega(x)=-\log |f(x)|=\sum_{k}-\left[2^{k} \delta\right] \log \left|\cos \left(\frac{x}{2^{k}}\right)\right| \geq-\left[2^{N} \delta\right] \log \left|\cos \left(\frac{x}{2^{N}}\right)\right|, \quad x \in \mathbb{R} .
$$

We return to a lower estimate for $\Omega$ on $I_{N}:=\left[x_{N}-1, x_{N}+1\right]$ :

$$
\begin{aligned}
\Omega(x) & \geq-\left[2^{N} \delta_{N}\right] \log \left|\cos \left(\frac{x}{2^{N}}\right)\right| \geq-\left[2^{N} \delta_{N}\right] \log \left(\cos \left(\frac{\frac{\pi}{2} 2^{N}+1}{2^{N}}\right)\right) \\
& =\left[2^{N} \delta_{N}\right]\left|\log \left(\sin \left(1 / 2^{N}\right)\right)\right| \geq\left(2^{N} \delta_{N}-1\right) \log \left(2^{N}\right) \\
& \geq \frac{2}{\pi} \delta_{N} x_{N} \log x_{N}\left[1+\frac{\log (2 / \pi)}{\log x_{N}}-\frac{1}{\delta_{N} x_{N}}\right] \\
& \geq\left(\frac{2}{\pi} C \gamma\left(x_{N}\right) x_{N} \log x_{N}\right) \frac{1}{4} \geq \gamma\left(x_{N}\right) x_{N} \log x_{N}
\end{aligned}
$$

if $x \in I_{N}, N \in \mathbb{N}^{*}, \min \mathbb{N}^{*}$, and $C$ are sufficiently large. Consequently,

$$
S_{1}(\Omega)\left(x_{N}\right) \leq \gamma\left(x_{N}\right) x_{N} \log x_{N}
$$

for $N \in \mathbb{N}^{*}$, and it remains to choose $\mathbb{N}^{*}$ so sparse that $C \sum_{n \in \mathbb{N}^{*}} \gamma\left(x_{n}\right)<\sigma$.

Two statement that follow show that Theorem 4 forbids the existence of some majorants with moderately oscillating derivative and with convergent logarithmic integral $\mathcal{L}(\omega)$. 
Statement 5. For any monotone increasing positive function $M$ with $\lim _{x \rightarrow+\infty} M(x)=$ $+\infty$, there exists a positive bounded function $\omega=e^{-\Omega}$ such that
a) $\mathcal{L}(\omega)>-\infty$
b) $\left|\Omega^{\prime}(x)\right| \leq M(x)\left|\log ^{2}\right| x||$;
c) $\omega \notin \bigcup_{\sigma>0} \operatorname{Adm}\left(e^{i \sigma z}\right)$.

It is well known that if $\omega$ satisfies a) and is Lipschitz, then $\omega \in \bigcap_{\sigma>0} \operatorname{Adm}\left(e^{i \sigma z}\right)$.

Proof. There is no loss of generality in assuming that $2 \leq M(x) \leq x, x>10$. We put $l_{x}=\frac{2 x}{\sqrt{M(x)} \log (x)}(x>1000)$ and observe that $2<l_{x}<x / 5$. For every $x>1000$, we introduce the function $\Omega_{x}$ in the following way:

$$
\begin{gathered}
\operatorname{supp}\left(\Omega_{x}\right)=\left[x-l_{x}, x+l_{x}\right], \quad \Omega_{x}(x)=x(M(x))^{\frac{1}{3}} \log (x), \\
\Omega_{x} \text { is linear on }\left[x-l_{x}, x\right] \text { and }\left[x, x+l_{x}\right] .
\end{gathered}
$$

A direct calculation shows that

$$
\int_{\mathbb{R}} \Omega_{x}(t) d \mathbf{P}(t) \leq 100(M(x))^{-\frac{1}{6}} .
$$

The right-hand side of this inequality tends to zero. Put $j_{x}:=\left[x-l_{x}, x+l_{x}\right]$. Without loss of generality, we may assume that $(M(t)-M(s))<1 / 2$ for $t, s \in j_{x}$. It is easily seen that

$$
\left|\Omega_{x}^{\prime}(t)\right| \leq \frac{\Omega_{x}(x)}{l_{x}}=\frac{1}{2} M(x)^{\frac{5}{6}} \log ^{2}(x), \quad t \neq x-l_{x}, x, x+l_{x} .
$$

Consider a positive sequence $\left\{x_{n}\right\}_{n \in \mathbb{N}}$ such that the intervals $j_{x_{n}}$ are disjoint and the series $\sum_{n \in \mathbb{N}}\left(M\left(x_{n}\right)\right)^{-\frac{1}{6}}$ converges. We put

$$
\Omega=\sum_{n \in \mathbb{N}} \Omega_{x_{n}}
$$

Note that for $t \in j_{x_{n}}$ we have

$$
\begin{array}{r}
\left|\Omega^{\prime}(t)\right|=\frac{1}{2} M\left(x_{n}\right)^{\frac{5}{6}} \log ^{2}\left(x_{n}\right) \leq \frac{1}{2} M\left(x_{n}\right) \log ^{2}\left(x_{n}\right) \leq M(t)\left|\log ^{2}(t)\right|, \\
t
\end{array}
$$

(Smoothing out $\Omega$ near the points $x_{n}-l_{x_{n}}, x_{n}$, and $x_{n}+l_{x_{n}}$, we may assume that the inequality is fulfilled for all $t \in j_{x_{n}}$.) Clearly, $\omega=e^{-\Omega}$ satisfies a) and b). On the other hand,

$$
S_{1}(\Omega)\left(x_{n}\right) \geq \Omega\left(x_{n}+1\right) \geq \Omega\left(x_{n}+\frac{1}{2} l_{x_{n}}\right)=\frac{1}{2}\left(M\left(x_{n}\right)\right)^{\frac{1}{3}} x_{n} \log \left(x_{n}\right),
$$

which contradicts Example 1.

Statement 6. Let $B_{\alpha}$ be the Blaschke product with the zeros $z_{k}=\operatorname{sgn}(k)|k|^{\alpha}+i, 1 / 2<$ $\alpha<1$. Then for every positive monotone increasing function $M$ with $\lim _{x \rightarrow+\infty} M(x)=$ $+\infty$, there exists a nonnegative bounded function $\omega$ such that
a) $\mathcal{L}(\omega)>-\infty$;
b) $\left|\Omega^{\prime}(x)\right| \leq M(x)|x|^{2\left(\frac{1}{\alpha}-1\right)} \log ^{2}|x|$;
c) $\omega \notin \operatorname{Adm}\left(B_{\alpha}\right)$. with

On the other hand, in B12 it was proved that an arbitrary bounded function $\omega=e^{-\Omega}$
a) $\mathcal{L}(\omega)>-\infty$ and
b) $\left|\Omega^{\prime}(x)\right|=O\left(|x|^{\frac{1}{\alpha}-1}\right)$

is $B_{\alpha}$-admissible.

Proof. The arguments are much similar to those in the preceding example. 


\section{§6. Conditions sufficient for admissibility in the CAse of Blaschke PRODUCTS WITH TANGENT ARRANGEMENT OF ZEROS}

In this section, we consider Blaschke products with zeros $z_{k}=x_{k}+i y_{k}$ such that $y_{k} \in(0,1]$ (i.e., all zeros lie in the strip $\left.\{z \in \mathbb{C}: \operatorname{Im} z \in(0,1]\}\right)$ and $\lim _{k \rightarrow+\infty}\left|z_{k}\right|=+\infty$. The sequence $\left\{z_{k}\right\}$ satisfies the Blaschke condition, but we subject it to the even stronger condition

$$
\sum_{k \in \mathbb{Z}} \frac{1}{x_{k}^{2}}<+\infty .
$$

With every such Blaschke product $B$, we associate the Blaschke product $B_{1}$ with the zeros $x_{k}+i$ :

$$
B_{1}(z)=\prod_{k=-\infty}^{k=+\infty} e^{\beta_{k}} \frac{z-x_{k}-i}{z-x_{k}+i} .
$$

If the sequence $\left\{z_{k}\right\}$ satisfies (6.1), then the sequence $x_{k}+i$ satisfies the Blaschke condition, so that $B_{1}$ is well defined. All zeros of $B_{1}$ lie on the line $\mathbb{R}+i$. Conditions sufficient for $B_{1}$-admissibility can be found in $\mathrm{Bl} 2$. In this section, it will be convenient to assume that all majorants $\omega$ do not exceed 1 and belong to $L^{2}(\mathbb{R})$ (the definition of $\operatorname{Adm}(\Theta)$ is modified accordingly). We shall show that the classes $\operatorname{Adm}(\Theta)$ and $\operatorname{Adm}\left(\Theta_{1}\right)$ are closely related.

6.1. Lemma on products close to each other. In this subsection we prove $B$-admissibility criteria, including some cases where the zeros $\left\{z_{k}\right\}$ approach $\mathbb{R}$ tangentially as $k \rightarrow \pm \infty$. We compare the classes $\operatorname{Adm}(B)$ and $\operatorname{Adm}\left(B_{1}\right)$, the former being understood better. Comparison of $\operatorname{Adm}(\Theta)$ and $\operatorname{Adm}\left(\Theta_{1}\right)$ in the case where $\Theta_{1}$ is close to $\Theta$ in a sense was used in $[\mathrm{BH}$, Corollary 1.5, 1.6] and [BBH, Corollary 2.4].

The following quite simple fact is of the same sort. If $X$ is a set of functions and $F$ is a function, we shall denote by $X+F$ the set of all sums $x+F$, where $x \in X$.

We shall also need the following class of functions:

$$
\operatorname{ADM}(\Theta)=\{-\log \omega, \omega \in \operatorname{Adm}(\Theta)\}
$$

Lemma 4. Suppose that $\Theta$ and $\Theta_{1}$ are inner functions and

$$
\arg \Theta-\arg \Theta_{1}=2 h(D)+\text { const, }
$$

where $D \in L^{1}(\mathbf{P}), D \geq 0$. Then

$$
\operatorname{ADM}\left(\Theta_{1}\right) \cap\left\{F: F \in L^{1}(\mathbf{P}), F \geq D\right\}=\operatorname{ADM}(\Theta)+D .
$$

As usual, the symbol $h$ stands for the Hilbert transformation; here we deal with arbitrary inner functions, so that $\arg \Theta$ and $\arg \Theta_{1}$ are measurable branches of their argument. A real-valued function on $\mathbb{R}$ is said to be a good function (g.f.) if it is representable in the form $2 h(\log m)+2 \pi n+\gamma$, where $m \in L^{\infty}, m \geq 0, n$ is an integervalued function, and $\gamma \in \mathbb{R}$ (see Theorem 1 ).

Proof of Lemma 4. We shall write $\widetilde{M}$ in place of $h(M)$. Then $\arg \Theta_{1}+2(\widetilde{\Omega}+\widetilde{D})=$ $\arg \Theta+2 \widetilde{\Omega}+$ const is a g.f. by Theorem 1. Consequently, the set on the right in (6.4) is included in the set on the left. Now assume that $\Omega_{1} \geq D$ and $\Omega_{1} \in \operatorname{ADM}\left(\Theta_{1}\right)$. Then the nonnegative function $\Omega_{1}-D \geq 0$ belongs to $L^{1}(\mathbf{P})$. Using (6.3), we conclude that $\arg \Theta+2\left(\Omega_{1}-D\right)^{\sim}$ is a g.f. Consequently, $\Omega_{1}-D \in \operatorname{ADM}(\Theta)$. 
6.2. Applications. We are going to apply Lemma 4 with the specific pair $\left(\Theta, R_{\Theta}\right)$, where $\Theta=B$ is our initial Blaschke product with zeros $z_{k}=x_{k}+i y_{k}, 0<y_{k} \leq 1$, $\sum \frac{1}{x_{k}^{2}}<+\infty$, and

$$
R_{B}(x)=\frac{1}{2} \sum_{k \in \mathbb{Z}} \log \left(1+\frac{1-y_{k}^{2}}{\left(x-x_{k}\right)^{2}+y_{k}^{2}}\right) .
$$

We must verify (6.3) with $\Theta=B, \Theta_{1}=B_{1}$, and $R_{\Theta}=R_{B}$. We postpone this till the end of the section. For the moment, we take (6.3) for granted; that is, we adopt the formula

$$
\arg B-\arg B_{1}=2 \widetilde{R_{B}}+\text { const }
$$

(here $\arg B$ and $\arg B_{1}$ are understood as continuous arguments vanishing somewhere). We also postpone the verification of the convergence of the series in (6.5) and of the inclusion $R_{B} \in L^{1}(\mathbf{P})$ and turn directly to applications in the case of $x_{k} \equiv k$.

Corollary 3. Let $B$ be a Blaschke product with zeros $z_{k}=k+i y_{k} \quad\left(k \in \mathbb{Z}, y_{k} \leq 1\right)$. If $\omega=e^{-\Omega} \in L^{2}(\mathbb{R})$ and $\Omega \in \operatorname{Lip}_{1}(\mathbb{R}) \cap L^{1}(\mathbf{P})$, then $\Omega+R_{B} \in \operatorname{ADM}(B)$, i.e., $\omega=e^{-\bar{\Omega}-R_{B}} \in$ $\operatorname{Adm}(B)$.

Proof. The results of $[\mathrm{BH}]$, Corollary 2, and Example 1 show that the Beurling-Malliavin theorem is applicable to the class $\operatorname{Adm}\left(B_{1}\right)$ (here $B_{1}$ is the Blaschke product whose zeros are $k+i, k \in \mathbb{Z})$. Thus, $\Omega$ belongs to $\operatorname{ADM}\left(B_{1}\right)$. Consequently, $\Omega+R_{B} \in \operatorname{ADM}(B)$ by Lemma 4.

The next statement provides admissibility criteria in explicit terms.

Corollary 4. Let $B$ be a Blaschke product whose zeros are $z_{k}=k+i y_{k}\left(k \in \mathbb{Z}, y_{k} \leq 1\right)$. Suppose that the two-sided sequence $\left\{y_{k}\right\}$ is monotone decreasing for $k>0$, is even, and satisfies

$$
\sum_{k} \frac{\log y_{k}}{k^{2}}>-\infty .
$$

Then an arbitrary even function $\omega \in L^{2}(\mathbb{R})$ monotone decreasing on the ray $(0,+\infty)$ and satisfying $\mathcal{L}(\omega)>-\infty$ is $B$-admissible.

Proof. Let $k$ be an integer nearest to $x$. Then

$$
\begin{aligned}
R_{B}(x) & =\sum_{n \in \mathbb{Z}} \log \left(1+\frac{1-y_{n}^{2}}{(x-n)^{2}+y_{n}^{2}}\right) \\
& \leq \log \left(1+\frac{1}{y_{k}^{2}}\right)+\sum_{n \in \mathbb{Z}, n \neq k} \log \left(1+\frac{1-y_{n}^{2}}{(x-n)^{2}+y_{n}^{2}}\right) \\
& \leq 2\left|\log y_{k}\right|+\log 2+\sum_{n \neq k} \frac{1}{(x-n)^{2}} \leq 2\left|\log y_{n}\right|+5 .
\end{aligned}
$$

Put $R_{B}^{+}(x)=2\left|\log y_{k}\right|+5$, where $k$ is an integer nearest to $|x|$. It is easily seen that the function $R_{B}^{+} \geq R_{B}$ is even and monotone increasing on the ray $(0,+\infty)$. Consequently, the function $\Omega+R_{B}^{+}$is even and monotone increasing on $(0,+\infty)$ and $\int_{\mathbb{R}}\left(\Omega+R_{B}^{+}\right) d \mathbf{P}<$ $+\infty$. Therefore, $e^{-\left(\Omega+R_{B}^{+}\right)} \in \operatorname{Adm}\left(B_{1}\right)$ (we remind the reader that $B_{1}$ is the Blaschke product whose zeros are $k+i, k \in \mathbb{Z})$. Lemma 4 shows that $e^{-\left(\Omega+R_{B}^{+}-R_{B}\right)} \in \operatorname{Adm}(B)$. But $\Omega+R_{B}^{+}-R_{B} \geq \Omega$. Thus, $\omega=e^{-\Omega} \in \operatorname{Adm}(B)$. 
This result was obtained in $[\mathrm{BBH}]$ by a different method (the main difference is in the way of constructing an element of $K_{B}$ subordinate to $\omega$ ). Our approach proves to be more direct. It is also applicable to other Blaschke products (with zeros $\left\{z_{k}\right\}$ as above, but with oscillating $y_{k}$ ). This is supported by the following result.

Corollary 5. Let $B$ be the Blaschke product whose zeros are $z_{k}=k+i y_{k}(k \in \mathbb{Z}$, $\left.y_{k} \leq 1\right)$. Suppose that the quantities $y_{k} / y_{k+1}$ are bounded and bounded away from zero, and

$$
\sum_{k} \frac{\log y_{k}}{k^{2}}>-\infty
$$

Then an arbitrary function $\omega=e^{-\Omega} \in L^{2}(\mathbb{R})$ with $\Omega \in \operatorname{Lip}_{1}(\mathbb{R}) \cap L^{1}(\mathbf{P})$ is B-admissible.

Proof. Put $R_{B}^{+}(x)=2\left|\log y_{k}\right|+5$, where $k$ is an integer nearest to $x$, and observe that $R_{B}^{+} \geq R_{B}$ (see the proof of Corollary 4 ). But || $\log y_{k}|-| \log y_{k+1}|| \preceq 1$, and we can choose a constant $C$ such that $S_{1}\left(R_{B}^{+}\right)+C>R_{B}$ ( $S_{1}$ is the Steklov averaging operator). The function $\Omega+S_{1}\left(R_{B}^{+}\right)+C$ is Lipschitz, and $\int_{\mathbb{R}}\left(\Omega+S_{1}\left(R_{B}^{+}\right)+C\right) d \mathbf{P}<+\infty$. Consequently, $e^{-\left(\Omega+S_{1}\left(R_{B}^{+}\right)+C\right)} \in \operatorname{Adm}\left(B_{1}\right)$. From Lemma 4 it follows that $e^{-\left(\Omega+S_{1}\left(R_{B}^{+}\right)+C-R_{B}\right)}$ belongs to $\operatorname{Adm}(B)$. Since $\Omega+S_{1}\left(R_{B}^{+}\right)+C-R_{B} \geq \Omega$, we see that $\omega=e^{-\Omega} \in \operatorname{Adm}(B)$.

We observe that, for such $B$, an arbitrary even positive function monotone decreasing on $(0,+\infty)$ belongs to $\operatorname{Adm}(B)$; see [HJ].

6.3. The function $R_{B}$ : Proof of formula (6.6) . First, we prove that the sum defining $R_{B}$ converges:

$$
\sum_{k} \log \left(1+\frac{1-y_{k}^{2}}{\left(x-x_{k}\right)^{2}+y_{k}^{2}}\right) \leq \sum_{k} \frac{1-y_{k}^{2}}{\left(x-x_{k}\right)^{2}+y_{k}^{2}}
$$

and the series converges uniformly on any compact set. Now, we show that $R_{B}$ is integrable against the Poisson measure. We calculate the contribution of each term of the series to the Poisson integral. The function $w(z):=\frac{z-x_{n}+i}{z-x_{n}+i y_{n}}$ is analytic and does not vanish on $\mathbb{C}^{+}$. The function $\log |w|$ is harmonic and bounded on $\mathbb{C}^{+}$:

$$
\begin{aligned}
\int_{\mathbb{R}} \log \left(1+\frac{1-y_{n}^{2}}{\left(x-x_{n}\right)^{2}+y_{n}^{2}}\right) d \mathbf{P} & =2 \pi \log \left|\frac{i-x_{n}+i}{i-x_{n}+i y_{n}}\right| \\
& =\pi \log \frac{4+x_{n}^{2}}{\left(1+y_{n}\right)^{2}+x_{n}^{2}} \leq \pi \log \frac{4+x_{n}^{2}}{2+x_{n}^{2}} \leq \frac{\pi}{3+x_{n}^{2}} .
\end{aligned}
$$

Consequently,

$$
\int_{\mathbb{R}} R_{B} d \mathbf{P}=\sum_{n} \int_{\mathbb{R}} \log \left(1+\frac{1-y_{n}^{2}}{\left(x-x_{n}\right)^{2}+y_{n}^{2}}\right) d \mathbf{P} \leq \sum_{n} \frac{\pi}{3+x_{n}^{2}}<+\infty .
$$

We prove (6.6). For every $z \in \mathbb{C}^{+}, z=x+i y$, put $L_{z}(t):=\log \frac{(t-x)^{2}+1}{(t-x)^{2}+y^{2}}, t \in \mathbb{R}$. Formula (6.5) implies

$$
h\left(R_{B}\right)(t)=\sum_{k} h\left(L_{z_{k}}\right)(t) .
$$

The series converges uniformly on any compact set because $\sum_{k} \frac{1}{x_{k}^{2}}<+\infty$. If we prove that

$$
\left[h\left(R_{B}\right)\right]^{\prime}=(\arg B)^{\prime}-\left(\arg B_{1}\right)^{\prime}
$$


everywhere on $\mathbb{R}$, then (6.6) will follow. To prepare the proof of (6.8), we introduce yet another version $h_{0}$ of the Hilbert transformation:

$$
h_{0}(f)(x)=\frac{1}{\pi} \mathrm{P} \cdot \mathrm{V} \cdot \int_{\mathbb{R}} \frac{f(t)}{x-t} d t .
$$

The operator $h_{0}$ is well defined for every $f \in L^{1}\left(\mathbf{P}_{0}\right)$, where $d \mathbf{P}_{0}(t)=d t /(1+|t|)$; the right-hand side in (6.9) makes sense for a.e. $x \in \mathbb{R}$. We observe that $h(f)=h_{0}(f)+$ const for such $f$. The operator $h_{0}$ commutes with translations and dilations (contractions) $x \mapsto$ $\lambda x$ of the real line $(\lambda>0)$; next, $h_{0}$ commutes with differentiation (if $f \in L^{1}\left(\mathbf{P}_{0}\right) \cap C^{1}(\mathbb{R})$ and $f^{\prime} \in L^{1}\left(\mathbf{P}_{0}\right)$, then $\left.h_{0}\left(f^{\prime}\right)=\left[h_{0}(f)\right]^{\prime}\right)$. Clearly,

$$
L_{z}^{\prime}(t)=2\left[\frac{t-x}{(t-x)^{2}+1}-\frac{t-x}{(t-x)^{2}+y^{2}}\right], \quad t \in \mathbb{R},
$$

so that $L_{z} \in L^{1}\left(\mathbf{P}_{0}\right), L_{z}^{\prime} \in L^{1}\left(\mathbf{P}_{0}\right)$. Therefore,

$$
\left[h\left(L_{z}\right)\right]^{\prime}(t)=\left[h_{0}\left(L_{z}\right)\right]^{\prime}(x)=\left[h_{0}\left(L_{z}^{\prime}\right)\right](t)=\frac{y}{(t-x)^{2}+y^{2}}-\frac{1}{(t-x)^{2}+1}, \quad x \in \mathbb{R} .
$$

The last identity follows from the properties of $h_{0}$ and the formula

$$
h_{0}\left(\frac{x}{1+x^{2}}\right)=-\frac{1}{1+x^{2}}
$$

(among numerous proofs, the shortest is probably obtained from the residue theorem). To obtain (6.8), we differentiate the series in (6.7) (we have uniform convergence on compact sets). From (6.9) we deduce that

$$
[h(A)]^{\prime}(t)=\sum_{k} \frac{y_{k}}{\left(t-x_{k}\right)^{2}+y_{k}^{2}}-\frac{1}{\left(t-x_{k}\right)^{2}+1}=(\arg B)^{\prime}(t)-\left(\arg B_{1}\right)^{\prime}(t), \quad t \in \mathbb{R} .
$$

\section{REFERENCES}

[B] A. D. Baranov, Polynomials in the de Branges spaces of entire functions, Ark. Mat. 44 (2006), no. 1, 16-38. MR2237209 (2007m:46036)

[Ba] N. K. Bari, Trigonometric series, Fizmatgiz, Moscow, 1961; English transl., A treatise on trigonometric series. Vols. I, II, The Macmillan Co., New York, 1964. MR0126115 (23:A3411) MR.0171116 (30:1347)

[BB] A. D. Baranov and A. A. Borichev, Entire functions of exponential type with prescribed modulus on the real axis (unpublished).

[BBH] A. D. Baranov, A. A. Borichev, and V. P. Havin, Majorants of meromorphic functions with fixed poles, Indiana Univ. Math. J. 56 (2007), no. 4, 1595-1628. MR 2354693 (2008i:30034)

[BH] A. D. Baranov and V. P. Khavin, Admissible majorants for model subspaces and arguments of inner functions, Funktsional. Anal. i Prilozhen. 40 (2006), no. 4, 3-21; English transl., Funct. Anal. Appl. 40 (2006), no. 4, 249-263. MR2307699(2008c:30058)

[B11] Yu. S. Belov and V. P. Khavin, On a theorem of I. I. Privalov on the Hilbert transform of Lipschitz functions, Mat. Fiz. Anal. Geom. 11 (2004), no. 4, 380-407. (Russian) MR2114001 (2005k:26006)

[B12] Yu. S. Belov, Admissibility criteria for model subspaces with fast growth of the argument of the generating inner function, Zap. Nauchn. Sem. S.-Peterburg. Otdel. Mat. Inst. Steklov. (POMI) 345 (2007), 55-84; English transl., J. Math. Sci. (New York) 148 (2008), no. 6, 813-829. MR2432176

[B13] Model functions with nearly prescribed modulus, Algebra i Analiz 20 (2008), no. 2, 3-18; English transl., St. Petersburg Math. J. 20 (2009), 163-174. MR2423994 (2009e:30079)

[D] K. M. D'yakonov, Moduli and arguments of analytic functions from subspaces in $H^{p}$ that are invariant under the backward shift operator, Sibirsk. Mat. Zh. 31 (1990), no. 6, 64-79; English transl., Siberian Math. J. 31 (1990), no. 6, 926-939 (1991). MR1097956 (92f:30049)

[HJ] V. Havin and B. Jöricke, The uncertainty principle in harmonic analysis, Ergeb. Math. Grenzgeb. (3), Bd. 28, Springer-Verlag, Berlin, 1994. MR1303780 (96c:42001) 
[HM1] V. P. Havin and J. Mashreghi, Admissible majorants for model subspaces of $H^{2}$. I. Slow winding of the generating inner function, Canad. J. Math. 55 (2003), no. 6, 1231-1263. MR2016246 (2004i:30029a)

[HM2] Admissible majorants for model subspaces of $H^{2}$. II. Fast winding of the generating inner function, Canad. J. Math. 55 (2003), no. 6, 1264-1301. MR2016247(2004i:30029b)

[MNH] J. Mashreghi, F. L. Nazarov, and V. P. Khavin, The Beurling-Malliavin multiplier theorem: The seventh proof, Algebra i Analiz 17 (2005), no. 5, 3-68; English transl., St. Petersburg Math. J. 17 (2006), no. 5, 699-744. MR2241422 (2007g:42028)

[N] N. K. Nikol'skiı̆, Treatise on the shift operator, "Nauka", Moscow, 1980; English transl., Grundlehren Math. Wiss., Bd. 273, Springer-Verlag, Berlin, 1986. MR0575166|(82i:47013) MR.0827223 (87i:47042)

Department of Mathematics and Mechanics, St. Petersburg State University, UniversitetSkil Prospekt 20, Petrodvorets, 198504 St. Petersburg, Russia

E-mail address: j_b_juri_belov@mail.ru

Received 20/FEB/2008

Translated by S. KISLYAKOV 